\title{
Effects of Maturation on Cyclic GMP-Dependent Vasodilation in Ovine Basilar and Carotid Arteries
}

\author{
WILLIAM J. PEARCE, ANDREW D. HULL, DON M. LONG, AND CHARLES R. WHITE \\ Division of Perinatal Biology, Department of Physiology, Loma Linda University School of \\ Medicine, Loma Linda, California 92350
}

\section{ABSTRACT}

\begin{abstract}
The present experiments examine the effects of maturation on cyclic GMP (cGMP)-mediated vasodilation in 688 segments of common carotid (COM) and basilar (BAS) arteries taken from newborn (3- to 7-d-old) and nonpregnant adult sheep. The main finding is that maximum efficacy for relaxation decreased with maturation in both artery types for the nitric oxide releasing vasodilators $\mathrm{S}$-nitroso-N-acetyl-penicillamine and nitroglycerin. These decreases could not be explained by changes in the - log $\mathrm{ED}_{50}$ concentrations for either vasodilator. Determination of the time course of cGMP responses to S-nitroso-Nacetyl-penicillamine or nitroglycerin at $10 \mu \mathrm{M}$ revealed that the peak cGMP responses to these agents (range: $5.3 \pm 0.8$ to $8.3 \pm 1.6 \mathrm{pmol} / \mathrm{mg}$ of protein) also did not vary significantly with age. However, cGMP attained peak values more rapidly in adult (COM: $50 \mathrm{~s}$; BAS $30 \mathrm{~s}$ ) than in newborn (COM: 60-80 s: BAS, 40-60 s) segments and returned to baseline more slowly in newborn than in adult segments, suggesting that maturation accelerates cGMP turnover. Correspondingly, baseline levels of cGMP were
\end{abstract}

In most arteries, vascular structure and function are immature at birth and change markedly during early postnatal life (1). In the cerebral circulation, this immaturity may be particularly important, because it may contribute to the high incidence of cerebrovascular complications observed in neonates $(2,3)$. Among the few studies that have directly examined maturational changes in cerebral arteries, however, most have found that vasoconstrictor reactivity is generally higher in immature than in mature cerebral arteries $(1,4-6)$. Thus, the high incidence of neonatal cerebrovascular complications seems not to be a consequence of a deficit in the ability of cerebral arteries to respond to vasoconstrictor stimuli. Alternatively, it remains possible that altered mechanisms of cerebral vasorelaxation may be involved. If

Received November 30, 1993; accepted February 23, 1994.

Correspondence: William J. Pearce, Ph.D., Division of Perinatal Biology, Loma Linda University School of Medicine, Loma Linda, CA 92350.

Supported by PHS Grant HLA1347 and Grant 92-293A from the American Heart Association. higher in newborn (COM: $1.0 \pm 0.1$; BAS: $3.3 \pm 0.5$ $\mathrm{pmol} / \mathrm{mg}$ of protein) than in adult (COM: $0.3 \pm 0.1$; BAS: $1.7 \pm 0.2 \mathrm{pmol} / \mathrm{mg}$ of protein) segments. Despite these differences in cGMP time course, rates of relaxation in response to $\mathrm{S}$-nitroso- $\mathrm{N}$-acetyl-penicillamine and nitroglycerin did not vary significantly with age, indicating that the temporal relation between cGMP and relaxation is different in newborn and adult arteries. Together, these results suggest that the capacity of the cGMP pathway to produce relaxation is attenuated by maturation through changes possibly related to cGMP turnover. (Pediatr Res 36: 25-33, 1994)

Abbreviations
ANOVA, analysis of variance
cGMP, cyclic GMP
pD, the negative logarithm of the concentration of an
agent that produces half its maximal effect
SNAP, S-nitroso-N-acetyl-penicillamine
NG, nitroglycerin

newborn arteries have a greater propensity to relax, they may be less capable of resisting the vascular injury and rupture often associated with insults, such as asphyxia and transient hypertension, that markedly elevate cerebral perfusion. Mechanisms of cerebral vasorelaxation, however, have thus far received relatively little direct experimental attention in neonates.

One of the most important pathways for vasorelaxation is that mediated by changes in vascular guanylate cyclase activity. Increases in cGMP synthesis mediate the vasodilatory responses to nitrovasodilators, atrial natriuretic peptide, and also nitric oxide released from either perivascular neurons or vascular endothelial cells (7-9). Recent evidence further suggests that, in adults of some species, changes in cGMP may also be involved in cerebrovascular responses to hypercapnia (10) and possibly also hypoxia $(11,12)$. Other studies suggest that the sensitivity of guanylate cyclase to nitric oxide may change during maturation, at least in the pulmonary circulation (13). Given the central importance of the guanyl- 
ate cyclase-cGMP pathway, its potential modulation by maturation, and the fact that it has been little studied in the cerebral circulation, the present studies address the hypothesis that maturation modulates the cGMP pathway for relaxation in cerebral arteries.

To explore this hypothesis, we have examined and compared cGMP-dependent, but endothelium-independent, relaxation in arteries isolated from newborn and adult sheep. Given previous suggestions of important intracranial-extracranial differences in patterns of relaxation in this model $(14,15)$, we studied both common carotid and basilar arteries. In light of suggestions that maturation may alter the capacity for biotransformation of NG (14), we examined two cGMP-dependent vasodilators: NG, which requires intracellular biotransformation for its action, and S-nitroso- $\mathrm{N}$-acetyl-penicillamine, which does not. For both of these agents, we examined the effects of maturation on their dose-response relations and on the dynamic relations between relaxation and cGMP synthesis.

\section{METHODS}

All procedures and protocols used in the present studies were approved by the Animal Research Committee of Loma Linda University and followed all guidelines put forth in the NIH Guide for the Care and Use of Laboratory Animals.

We obtained basilar and common carotid arteries from newborn lambs (aged 3-5 d) and young, nonpregnant adult sheep (aged 18-24 mo) killed by pentobarbital overdose. Up to eight ring segments of each artery type were sampled from each animal. From 20 newborn lambs, we harvested 240 artery segments, and from 28 adult sheep, we harvested a total of 448 . When a single protocol was repeated with multiple segments from the same animal, we averaged the results into a single value before statistical analysis. All reported values of $n$ refer to the number of animals, not the number of segments.

The initial treatment of all arteries was identical and has been described in detail previously (1). Briefly, we cleaned the arteries of adipose and connective tissue and cut them into ring segments $3 \mathrm{~mm}$ in length. All arteries were denuded of vascular endothelium by mild mechanical abrasion, after which we mounted each vascular ring on paired wires between a force transducer (BG-10, Kulite Semiconductors, Ridgefield, NJ) and a post attached to a micrometer used to vary resting tension. In a Krebsbicarbonate solution containing (in $\mathrm{mM}$ ) $122 \mathrm{NaCl}, 25.6$ $\mathrm{NaHCO}_{3}, 5.56$ dextrose, $5.17 \mathrm{KCl}, 2.49 \mathrm{MgSO}_{4}, 1.60$ $\mathrm{CaCl}_{2}, 0.114$ ascorbic acid, and 0.027 disodium EDTA, continuously bubbled with $95 \% \mathrm{O}_{2}$ and $5 \% \mathrm{CO}_{2}$ and maintained at $38.5^{\circ} \mathrm{C}$ (normal ovine core temperature), we slowly and repeatedly stretched freshly mounted arteries until optimum baseline tensions of $1 \mathrm{~g}$ for the common carotid and $0.5 \mathrm{~g}$ for the basilar arteries (1) remained stable for at least $30 \mathrm{~min}$. We then contracted the arteries with an isotonic potassium Krebs solution containing $122 \mathrm{mM} \mathrm{K}{ }^{+}$and $31 \mathrm{mM} \mathrm{Na}{ }^{+}$. After peak tensions were reached, we washed the arteries with normal sodium Krebs and allowed them to reequilibrate at baseline tension for another $30 \mathrm{~min}$. Endothelial denudation was then verified by the absence of a vasodilator response to the endothelium-dependent vasodilator A23187 (calcium ionophore, $1 \mu \mathrm{M}$ ) in precontracted arteries. Treatment of the artery segments thereafter varied with each protocol. During all experiments, we continuously digitized, normalized, and recorded contractile tensions using an on-line computer.

We conducted three experimental protocols. First, we determined the dose-response relations for the vasorelaxants SNAP and NG. Second, we determined the relaxation time courses for each agent at a dose of $10 \mu \mathrm{M}$. Finally, we determined the relations between the levels of the intracellular second messenger guanosine $3^{\prime}, 5^{\prime}$ cyclic monophosphate (cGMP) and the duration of exposure to SNAP and NG.

Dose-response experiments. Following the regimen described above, we induced a second contraction using a mixture of $20 \mu \mathrm{M}$ histamine with $10 \mu \mathrm{M}$ serotonin. Previous studies have shown this mixture to produce stable maximal contractions in ovine cerebral arteries (1). Once stable tone was established, we added cumulative doses of either SNAP or NG in aqueous solution to the tissue baths. Both agents were given in $1 / 2-\log$ increments to yield bath concentrations between $10^{-11}$ and $10^{-4} \mathrm{M}$. Because SNAP is somewhat unstable in solution, it was prepared immediately before use and held on ice. We calculated and expressed relaxant responses as the percentage of relaxation of the maximum initial tone attained in each vessel type. The maximum percentage of relaxation, $\mathrm{pD}_{2}$ value (negative $\log$ of the $\mathrm{ED}_{50}$ ), and Hill coefficient (related to slope) for each dose-response relation were determined by fitting the normalized doseresponse relation with the logistic equation using computerized nonlinear regression.

Vasorelaxation experiments. The artery segments used in our second protocol were also contracted with high potassium Krebs, washed, rested, and contracted with the mixture of serotonin and histamine as described above. After the establishment of stable contractile tone, we added either $10 \mu \mathrm{M}$ SNAP or $10 \mu \mathrm{M}$ NG to the baths and recorded the resulting changes in tension as a function of time. All relaxations were normalized relative to maximum initial contractile tension. Rates of relaxation were determined by fitting the relaxation time courses to a monoexponential decay model with a non-zero asymptote $\left(\mathrm{y}=\mathrm{Ae}^{-\mathrm{kt}}+\mathrm{B}\right)$ using computerized nonlinear regression, as previously described (16). In this model, $y$ is the percent initial tone remaining at time $t, A$ is the magnitude of maximum relaxation, $B$ is the asymptotic value of tone remaining at infinite time, and $k$ is the rate constant for relaxation.

Cyclic nucleotide responses. In arteries from a third group of animals, we determined the relations between cGMP and the duration of exposure to either $10 \mu \mathrm{M}$ 
SNAP or $10 \mu \mathrm{M}$ NG. We obtained a maximum of eight segments of each artery type from each animal, and all were studied simultaneously. We contracted the arteries with high potassium Krebs, washed them, and contracted them again with the mixture of serotonin and histamine as described above. After the establishment of stable tone, we froze one segment by rapid immersion in liquid nitrogen. This segment was used to determine the control levels of cGMP. We then added either $10 \mu \mathrm{M}$ SNAP or 10 $\mu \mathrm{M} N \mathrm{NG}$ to the remaining segments, which were then flash frozen at 10 -s intervals, up to a maximum of $100 \mathrm{~s}$ after relaxant addition. Thus, from each animal used in these experiments, we obtained a set of artery segments whose treatment varied only by the duration of exposure to the vasorelaxant used. To verify that the agents used produced no significant changes in cAMP, all samples were also routinely assayed for cAMP.

Cyclic nucleotide and protein determinations. Artery segments designated for cyclic nucleotide analysis were stored at $-80^{\circ} \mathrm{C}$ until assay, at which time they were individually homogenized in $1 \mathrm{~mL}$ of ice-cold $6 \%$ trichloroacetic acid using a motor-driven ground-glass pestle and mortar (Lurex, Vineland, NJ). After centrifuging the homogenates for $60 \mathrm{~min}$ at $3000 \times \mathrm{g}$, we reserved the resultant pellet for protein determination and decanted the supernatants for subsequent cyclic nucleotide assay. For protein content determinations, we used an extraction $\left(60 \mathrm{~min}\right.$ in $1.0 \mathrm{M} \mathrm{NaOH}$ at $\left.37^{\circ} \mathrm{C}\right)$ designed to exclude connective tissue and structural proteins, as previously described (1). We quantified protein using the Bradford Coomassie brilliant blue assay. As we have shown previously, this assay produces protein values that are both consistent and uniform in the vessel types studied (1).

For cyclic nucleotide determinations, we extracted the reserved supernatants with water-saturated diethyl ether, then lyophilized aliquots of the aqueous phase, reconstituted them in $50 \mathrm{mM}$ acetate buffer, and assayed for cyclic nucleotides using standard RIA techniques. We determined both cGMP and cAMP content for each sample using commercially available kits (RPA 525 and RPA 509, Amersham Corp., Arlington Heights, IL). Both cAMP and cGMP values were normalized relative to vessel protein content and were expressed as pmol per mg of vessel protein.

Assay validation. Given our interest in the relative effects of SNAP and NG on the levels of cGMP and cAMP and the possibility of interactions between these cyclic nucleotides, we evaluated the sensitivity and specificity of the assays used. Prior experience with commercially prepared cyclic nucleotide RIA kits indicated an unacceptable level of cross-reactivity between the antibodies for cGMP and cAMP provided with some kits. We determined specificity by overloading the assays for cAMP and cGMP with known amounts of cGMP $(7200 \mathrm{fmol})$ and cAMP (4800 fmol), respectively. In each case, under the conditions we used, the assays selected for this study failed to recognize ("undetectable") the added competing cyclic nucleotide. Standard curves for both assays covered the range of 1.5 to $96 \mathrm{fmol}$ per $75-\mu \mathrm{L}$ sample and thus encompassed the range of values of cGMP and cAMP seen in our samples. Therefore, the amounts of cGMP and cAMP added to test specificity represented considerable excesses over the levels normally seen.

Under our conditions, the assays we used consistently and reproducibly quantified low levels $(1.5 \mathrm{fmol}$ per $75-\mu \mathrm{L}$ sample) of both cyclic nucleotides in our samples. Intraassay duplicate errors were $6 \%$ or less, and interassay errors averaged less than $8 \% . \chi^{2}$ values for our standard curves were consistently close to 1.0 . The protein assay against which the cyclic nucleotide values were normalized routinely gave duplicate errors of $<5 \%$ and standard curve $r^{2}$ values of 0.995 or better. Thus, we believe that the values of cGMP and cAMP measured in this study were accurate and specific.

Statistics. All values were calculated as the mean \pm SEM. In all cases, $n$ refers to the number of animals used in a given experimental group. For all data sets, we evaluated the homogeneity of variance assumption among subsets (homoscedasticity) using Bartlett's test. Where homoscedasticity was verified across groups under the same treatment (SNAP or NG), we used either one-way or two-way ANOVA with maturational age (newborn or adult) as one factor and artery type (common carotid or basilar) as the other. From each ANOVA, there were up to three statistical results: 1) age effect; 2) artery effect; and 3 ) interaction effect. For ANOVA with one or more statistically significant results, we calculated individual post-hoc differences between treatments of a given vessel type using Duncan's multiple range test. For data sets where homoscedasticity was not observed, we performed single one-time comparisons between corresponding newborn and adult arteries using a Behren's Fisher analysis with pooled weighted variance. Unless otherwise indicated, statistical significance implies $p<$ 0.05 .

\section{RESULTS}

We performed three basic analyses. First, we compared results between ages for each artery-treatment combination. Second, we compared treatments (SNAP versus NG) between each age-artery combination. Finally, we compared between artery types within each age-treatment combination.

Dose-response experiments. The cumulative doseresponse relations for both SNAP and NG are shown in Figure 1, and the values of the coefficients obtained by fitting these data with the logistic equation are given in Figure 2. As indicated in the upper panels of Figure 2, maximum efficacy decreased significantly with age across all four artery-treatment groups. In the common carotid arteries, values of maximal relaxation averaged $95.4 \pm$ $1.6 \%$ (newborn SNAP), $61.0 \pm 4.6 \%$ (adult SNAP), 96.0 $\pm 1.0 \%$ (newborn NG), and 78.8 $\pm 5.2 \%$ (adult NG) of initial contractile tone. Corresponding basilar values were $100.0 \pm 0.6 \%, 71.4 \pm 3.3 \%, 99.0 \pm 0.7 \%$, and 78.5 

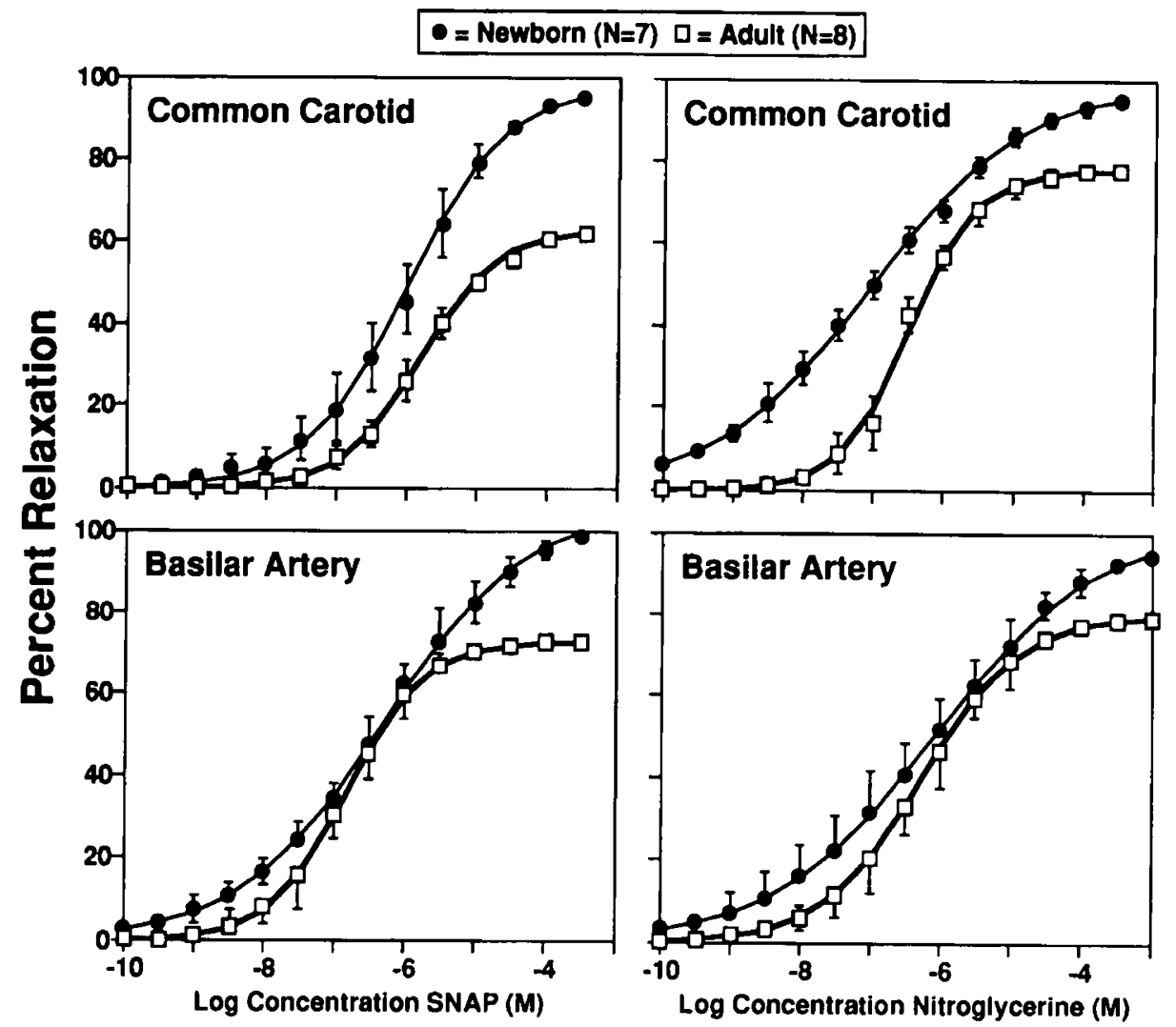

Figure 1. Cumulative dose responses to SNAP and NG were determined in arteries precontracted with $10 \mu \mathrm{M}$ serotonin and $20 \mu \mathrm{M}$ histamine. Average relaxant responses were calculated as the percentage of relaxation of initial contractile tone and are given for each artery type together with SEM where these exceed the size of the symbols. Arteries from seven newborn and eight adult animals were used.

$\pm 7.0 \%$. Maximum efficacy did not vary significantly with artery type and varied between corresponding SNAP. and NG-treated arteries only in the adult common carotid arteries.

Although $\mathrm{pD}_{2}$ values $\left(-\log \mathrm{ED}_{50}\right)$ tended to decrease with age in the common carotid and increase with age in the basilar arteries, $\mathrm{pD}_{2}$ changed significantly only for common carotid segments treated with NG (Fig. 2, middle panels). In the common carotid arteries, $\mathrm{pD}_{2}$ values averaged $6.08 \pm 0.29$ (newborn SNAP), $5.83 \pm 0.15$ (adult SNAP), $7.06 \pm 0.16$ (newborn NG), and $6.49 \pm$ 0.13 (adult NG). Corresponding basilar values were 6.39 $\pm 0.28,6.91 \pm 0.25,6.05 \pm 0.37$, and $6.30 \pm 0.23 . \mathrm{pD}_{2}$ values were significantly greater for NG than for SNAP in both newborn and adult common carotid segments. Significant differences between common carotid and basilar $\mathrm{pD}_{2}$ values occurred for SNAP in the adult and for NG in the newborn.

The slopes of the dose-response relations, as indicated by values of the Hill coefficient, increased significantly with age in arteries treated with NG (Fig. 2, lower pan$e l s)$. In the common carotid arteries, Hill values averaged $1.13 \pm 0.08$ (newborn SNAP), $1.09 \pm 0.06$ (adult SNAP), $0.88 \pm 0.05$ (newborn NG), and $1.18 \pm 0.07$ (adult NG). Corresponding basilar values were $0.92 \pm 0.06,1.25 \pm$ $0.19,0.80 \pm 0.04$, and $1.06 \pm 0.13$. Significant differences between Hill values for SNAP and NG occurred only in the newborn common carotid arteries. Significant differ- ences between common carotid and basilar Hill values were observed only for SNAP in the newborn.

Vasorelaxation time course. Relaxations to a single dose of either SNAP or NG were generally complete within $120 \mathrm{~s}$ in all arteries (Figs. 3 and 4). The rate coefficients for relaxation tended to decrease with age in the common carotid arteries and increase with age in the basilar arteries, but none of these differences were significant. Rate coefficients in the common carotid arteries averaged 1.06 \pm 0.14 (newborn SNAP), $0.82 \pm 0.08$ (adult SNAP), 1.17 \pm 0.20 (newborn NG), and $0.87 \pm 0.11$ (adult NG) per min. Corresponding basilar values were $2.19 \pm 0.33,2.85$ $\pm 0.39,1.37 \pm 0.28$, and $1.52 \pm 0.31$ per min. Rate values were significantly greater for SNAP than for NG only in adult basilar segments. Corresponding rate values were generally greater in basilar than in common carotid segments, and these differences were significant in all but newborn arteries treated with NG.

Cyclic nucleotide responses. cAMP responses were not significant in any artery of either age group treated with either SNAP or NG (Table 1). In contrast, control cGMP levels (at time zero in Figs. 3 and 4) were significantly greater in newborn than in adult arteries and averaged $1.04 \pm 0.14$ (newborn common carotid), $0.28 \pm 0.08$ (adult common carotid), $3.32 \pm 0.52$ (newborn basilar), and $1.68 \pm 0.23$ (adult basilar) $\mathrm{pmol} / \mathrm{mg}$ of protein. In addition, control cGMP levels were significantly lower in 

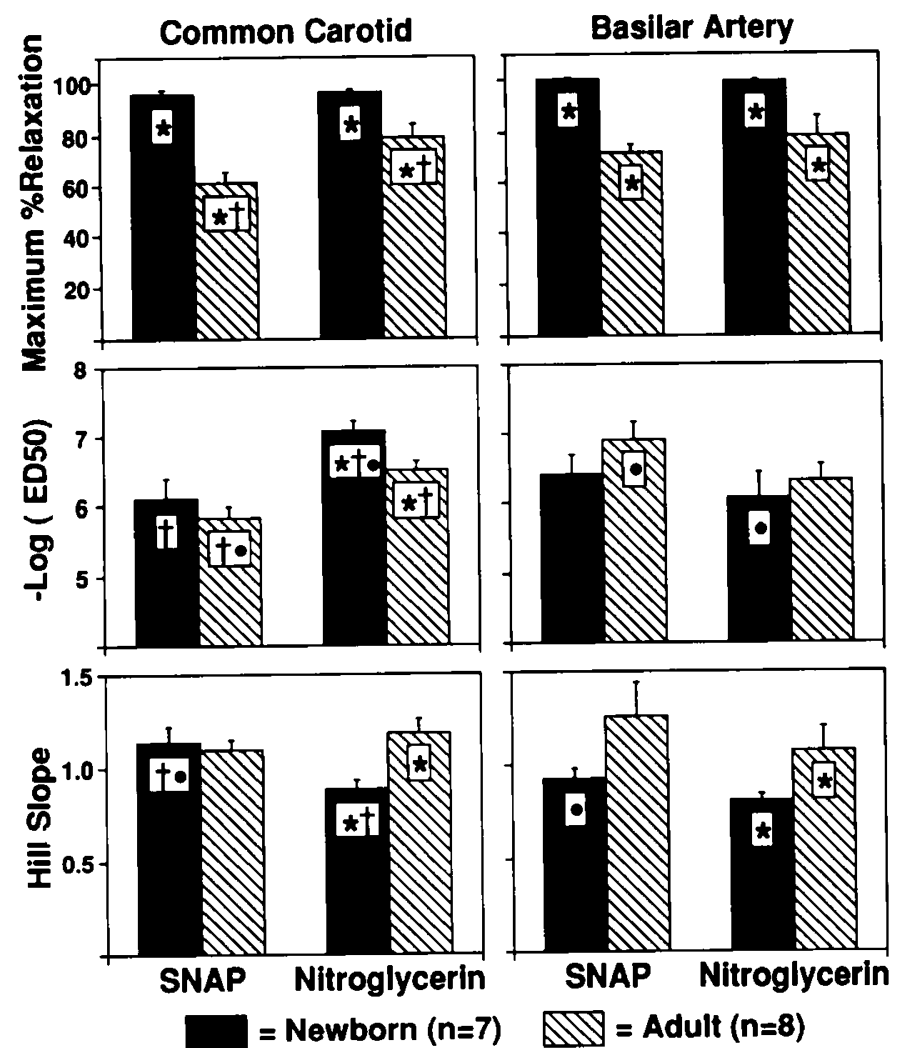

SNAP Nitroglycerin

Figure 2. The dose-response relations shown in Figure 1 were fitted to the logistic equation using nonlinear regression to obtain maximum percent relaxation values (upper panels) and the $\mathrm{pD}_{2}$ (negative log $\mathrm{ED}_{50}$ concentration, middle panels) and Hill slope (lower panels) coefficients for SNAP and NG for both artery types. All values are given as means \pm SEM for seven newborn and eight adult animals. The results of statistical comparisons between groups are given at the top of each panel: * indicates significant effects of age among arteries of the same type and treatment; $\dagger$ indicates significant differences between SNAP and NG for arteries of the same type and age; and ;ub indicates significant differences between common carotid and basilar arteries of the same age and treatment.

the common carotid than in the basilar arteries, in both newborns and adults.

In all arteries of both the newborn and the adult, administration of either SNAP or NG produced rapid increases in cGMP that peaked between 30 and $60 \mathrm{~s}$ and then returned toward baseline (Figs. 3 and 4). In all cases, peak cGMP values were attained earlier in adult arteries (common carotid: $50 \mathrm{~s}$; basilar: $30 \mathrm{~s}$ ) than in corresponding newborn arteries (common carotid: 60-80 s; basilar: $40-60 \mathrm{~s}$ ). In addition, in both artery types, newborn values were significantly higher than corresponding adult values at one or more points in time after adult peaks had been reached; the newborn time courses were shifted to the right relative to those of the adults.

Peak cGMP values tended to be greater in newborn than in adult arteries but varied significantly with age only in basilar segments treated with NG. In the common carotid arteries, peak values averaged $8.31 \pm 1.60$ (newborn SNAP), $5.92 \pm 0.78$ (adult SNAP), $5.56 \pm 1.44$ (newborn NG), and $5.33 \pm 0.82$ (adult NG) $\mathrm{pmol} / \mathrm{mg}$. Corresponding basilar values were $6.31 \pm 0.46,5.54 \pm$
$0.39,6.11 \pm 0.90$, and $3.97 \pm 0.61 \mathrm{pmol} / \mathrm{mg}$. Differences between peak values produced by SNAP and NG were significant only in adult basilar arteries. No significant differences between artery types were observed.

When values of percentage of relaxation observed at peak cGMP were compared, adult values were consistently lower than corresponding newborn values in all groups (Fig. 5, upper panels). In the common carotid arteries, these values averaged $79.3 \pm 2.4 \%$ (newborn SNAP), $45.2 \pm 5.5 \%$ (adult SNAP), $51.4 \pm 6.6 \%$ (newborn NG), and $29.9 \pm 4.0 \%$ (adult NG). Corresponding basilar values were $86.6 \pm 2.7 \%, 70.5 \pm 5.0 \%, 83.3 \pm$ $6.4 \%$, and $44.4 \pm 10.6 \%$. In addition, all values obtained with NG were lower than corresponding SNAP values, and these differences were significant in all but newborn basilar segments. Common carotid values were all lower than corresponding basilar values, and these differences were significant in all but adult arteries treated with NG.

\section{DISCUSSION}

The main finding of the present study is that maximum efficacy decreased with maturation for both SNAP- and NG-induced relaxation in common carotid and basilar arteries (Figs. 1 and 2). This result is consistent with previous suggestions that the mechanisms necessary for cGMP-dependent relaxation are fully functional in newborn arteries of the cerebral $(14,15)$ and pulmonary $(17$, 18) circulations. It also suggests that the maturational improvements in endothelium-dependent vasodilation reported in these vascular beds $(13,14,17)$ are more likely caused by functional changes in the endothelium than in the vascular smooth muscle of these arteries. From a clinical perspective, our results support the hypothesis that newborn cerebral arteries have a greater propensity to relax than corresponding adult arteries, at least in response to vasodilator stimuli dependent on guanylate cyclase activation. In turn, the greater tendency of newborn cerebral arteries to relax could contribute, at least theoretically, to the greater incidence of intracranial artery rupture observed in neonates relative to adults. (2, 3).

From a mechanistic perspective, the present results suggest that the capacity of the cGMP pathway to produce relaxation is attenuated by maturation. This attenuation does not seem to involve changes in the sensitivity to nitric oxide, because the $\mathrm{ED}_{50}$ concentrations for SNAP did not change with age (Fig. 2). Although the $\mathrm{ED}_{50}$ concentration for NG did increase slightly with age in the common carotid, this difference is probably secondary to maturational increases in wall thickness and thus diffusion distance, which are well documented in this artery (1). Whereas SNAP releases nitric oxide spontaneously upon hydration, NG must diffuse into the smooth muscle cells where it can be biotransformed to release nitric oxide $(19,20)$. Thus, the absence of an age-related change in sensitivity to SNAP in the common carotid artery may be attributed to the greater diffusabil- 


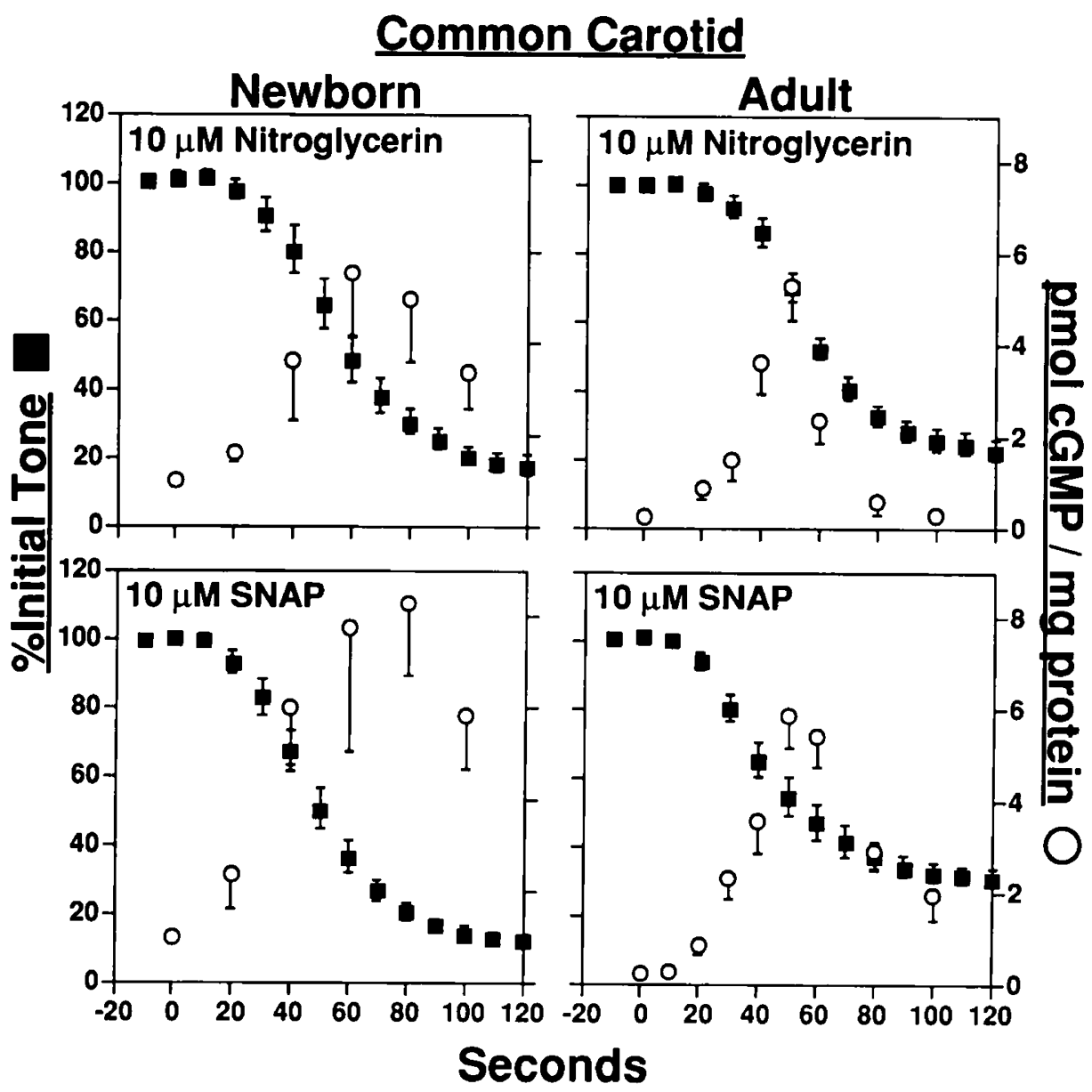

Figure 3. Newborn (left panels) and adult (right panels) common carotid arteries were precontracted with $10 \mu \mathrm{M}$ serotonin and $20 \mu \mathrm{M}$ histamine and then exposed to either $10 \mu \mathrm{M}$ NG (upper panels) or $10 \mu \mathrm{M}$ SNAP (lower panels), which rapidly liberates nitric oxide upon hydration. Relaxation responses are indicated by the filled squares and the cyclic nucleotide responses, measured by RIA in segments flash frozen 10 to 100 $\mathbf{S}$ after exposure to each relaxant, are shown by the open circles. All values are given as means and SEM. Artery segments from nine newborns and 12 adults were used to obtain the relaxation data. Segments from 11 newborns and 16 adults were used to obtain the cGMP data.

ity of nitric oxide released into solution by SNAP relative to the diffusability of the much larger NG molecule. This interpretation would also explain why age-related differences in sensitivity were not observed for either SNAP or NG in the relatively thin-walled basilar artery.

Another difference between responses to SNAP and NG was indicated by the Hill coefficient results. As calculated in the present study, the Hill coefficients quantify the slope of the relation between input (drug concentration) and output (relaxation response) and may be taken as a simple index of dose-response "gain." As such, the Hill coefficient results suggest that doseresponse gain increases with age for NG but not for SNAP. Because the mechanisms mediating relaxation subsequent to nitric oxide release are the same for SNAP and NG, the main difference between responses to these agents is that NG requires biotransformation and SNAP does not. Thus, we attribute the finding that the Hill coefficients increased significantly with age in both artery types for NG but not for SNAP to a maturational improvement in the capacity for the biotransformation of NG, as previously suggested (14).
The present results further suggest that the age-related decrease in the efficacy of SNAP and NG cannot be explained by a decrease in the peak amount of cGMP synthesized in response to a given concentration of nitric oxide. Peak cGMP levels produced by $10 \mu \mathrm{M}$ SNAP or NG were, in general, not significantly affected by maturation (Figs. 3 and 4). The time courses of the cGMP responses, however, were right-shifted in the newborn arteries, suggesting that the turnover of cGMP varied with age. Consistent with this interpretation, baseline cGMP levels were significantly higher in newborn than in adult arteries. These differences suggest that, relative to the rate for cGMP synthesis, the rate for cGMP breakdown may be higher in adult than in newborn arteries. Although intracellular cGMP concentration might also be reduced by extrusion from the cell, studies in adult rat aorta (21) suggest that the rate of cGMP extrusion is far slower than the rate of cGMP degradation by phosphodiesterase (22). Thus, age-related differences in cGMP turnover probably arise from differences in the relative rates of cGMP synthesis by guanylate cyclase or cGMP hydrolysis by phosphodiesterase. An important effect of 


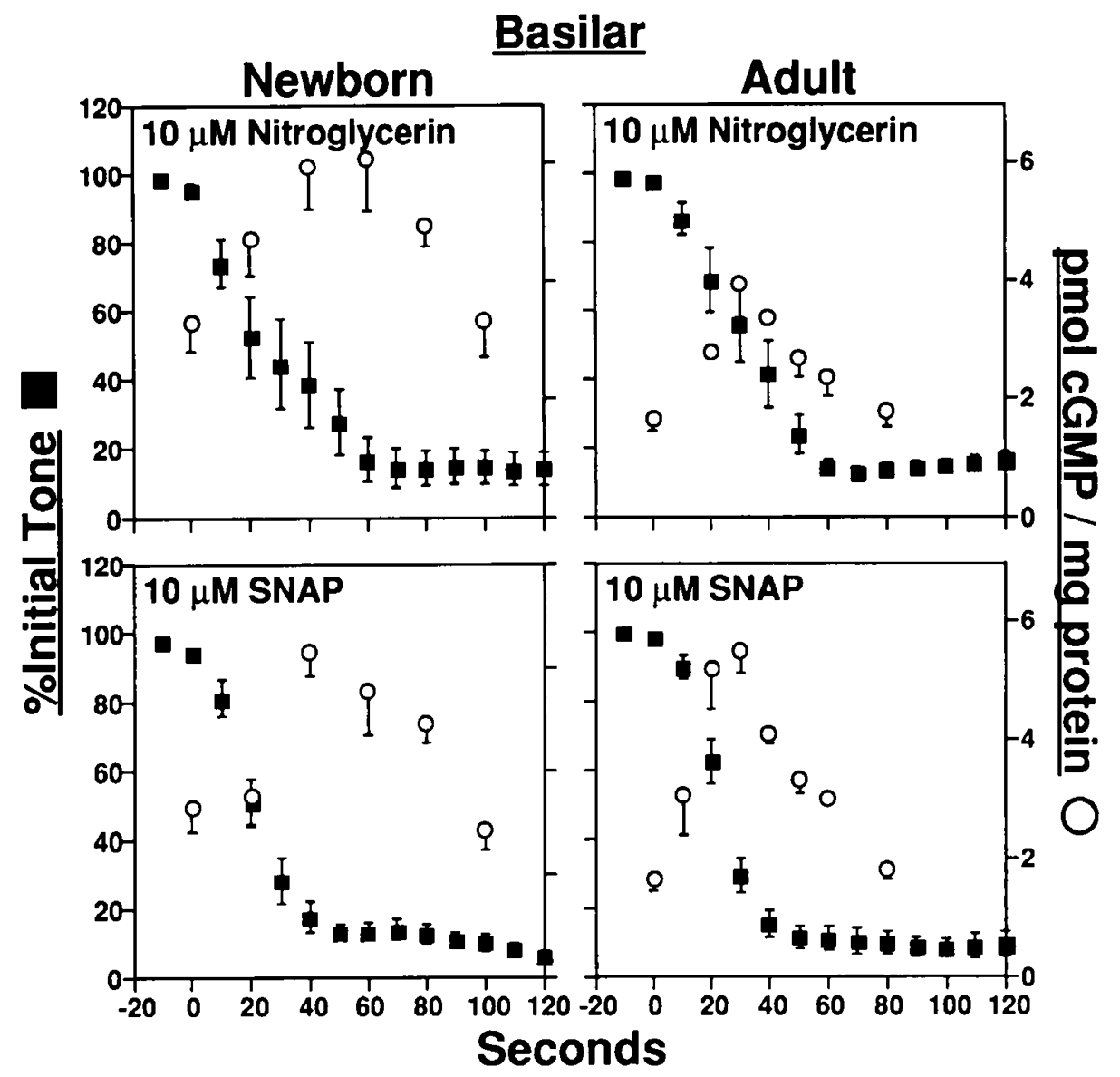

Figure 4. Shown here are data from the basilar artery corresponding to those for the common carotid artery shown in Figure 3. For details regarding symbols, abbreviations, and numbers of animals used, please refer to the legend for Figure 3.

Table 1. Effects of age and artery type on basal and peak cyclic nucleotide responses to NG and SNAP*

\begin{tabular}{cllcccc}
\hline Age & \multicolumn{1}{c}{ Artery } & Treatment & Baseline cGMP & Peak cGMP & Baseline cAMP & Peak cAMP \\
\hline Newborn & Common carotid & NG & $1.0 \pm 0.1$ & $5.6 \pm 1.4$ & $10.8 \pm 0.7$ & $11.4 \pm 0.8$ \\
& & SNAP & & $8.3 \pm 1.6$ & & $13.7 \pm 1.4$ \\
& Basilar & NG & $3.3 \pm 0.5$ & $6.1 \pm 0.9$ & $9.6 \pm 1.3$ & $8.5 \pm 1.0 \dagger$ \\
& & SNAP & & $5.6 \pm 0.9$ & & $6.5 \pm 0.4 \dagger$ \\
Adult & Common carotid & NG & $0.3 \pm 0.1$ & $5.3 \pm 0.8$ & $5.1 \pm 1.4$ & $4.0 \pm 0.9 \dagger$ \\
& & SNAP & & $5.9 \pm 0.8$ & & $6.6 \pm 1.1$ \\
& Basilar & NG & $1.7 \pm 0.2$ & $4.0 \pm 0.6$ & $5.1 \pm 0.6$ & $6.1 \pm 0.6$ \\
& & SNAP & & $5.5 \pm 0.6$ & & $5.4 \pm 0.6$ \\
\hline
\end{tabular}

${ }^{*}$ Given above are the baseline and peak cGMP values (in pmol/mg of protein) attained after treatment with either $10 \mu \mathrm{M}$ NG or $10 \mu \mathrm{M} \mathrm{SNAP}$. All values are given as the mean \pm SEM for 11 newborns and 16 adults. Baseline values were averaged across treatment groups; thus, a single value is shown. For comparison, corresponding cAMP values are also shown. Both SNAP and NG produced significant increases in cGMP, but neither agent significantly increased cAMP. The cGMP values given above are also plotted in Figures 3 and 4.

$\dagger$ In three cases, cAMP tended to decrease in response to either NG or SNAP, in which case the minimum values attained are shown.

such differences in cGMP turnover would be that the area beneath the cGMP-time curve would be greater for newborn than for adult arteries, as was observed.

By virtue of their effect on the area beneath the cGMPtime curve, maturational changes in cGMP turnover may at least partially explain the age-related decrease in maximum efficacy for SNAP and NG observed in the present experiments. Greater area beneath the cGMP-time curve should yield a greater total activation of G-kinase and greater phosphorylation of the proteins activated by G-kinase. This effect, in turn, could contribute to the greater relaxation observed in the newborn arteries. However, changes in the relation between cGMP concentration and relaxation may also be involved. The fact that relaxation persists long after cGMP has returned to baseline indicates that the duration of relaxation is determined by some process downstream from cGMP, possibly by smooth muscle phosphatase activity, which ultimately reverses the effect of G-kinase (23). Smooth muscle phosphatase activity could vary with age, although this possibility remains unexplored at present. Alternatively, the abundance or biochemical characteristics of the proteins phosphorylated by G-kinase might vary with age, which could potentially alter the relation between cGMP and 
relaxation as well as the maximum efficacy of nitric oxide. Given this range of possibilities, the present data can be taken to indicate only that a change in cGMP turnover probably plays some part in the maturational decease in the maximum efficacy for SNAP and NG.

One hint that maturation may change the relation between cGMP and relaxation is provided by the dynamic aspects of the present data. To examine these dynamics, we calculated the amount of relaxation attained when peak cGMP concentration was reached (Fig. 5, top panels). In all cases, relaxation was more complete in newborn than in adult arteries at peak cGMP. Rates of relaxation, however, did not vary significantly with age (Fig. 5 , bottom panels). These findings demonstrate that the dynamic relation between vascular cGMP and relaxation was different in newborn and adult arteries; although cGMP rose more slowly and was more sustained in the newborn arteries, their rate of relaxation was similar to that observed in the adult arteries. It remains possible, however, that the rate-limiting determinant of relaxation was similar in newborn and adult arteries. For example, if the initial rates of change in cGMP in these experiments were fast compared with the velocity of phosphorylation by G-kinase (24) or the physiologic effect of this phosphorylation $(25,26)$, these later steps may have governed relaxation rate (8). Similarly, if the cGMP changes in these experiments were supramaximal for activation of G-kinase, the cGMP time course need not bear any dynamic relation to relaxation rate. Clearly, many additional experiments will be required to determine why the cGMP time course changed with age but the rate of relaxation did not.

One final feature of the present results worthy of note concerns the modest differences observed between com-
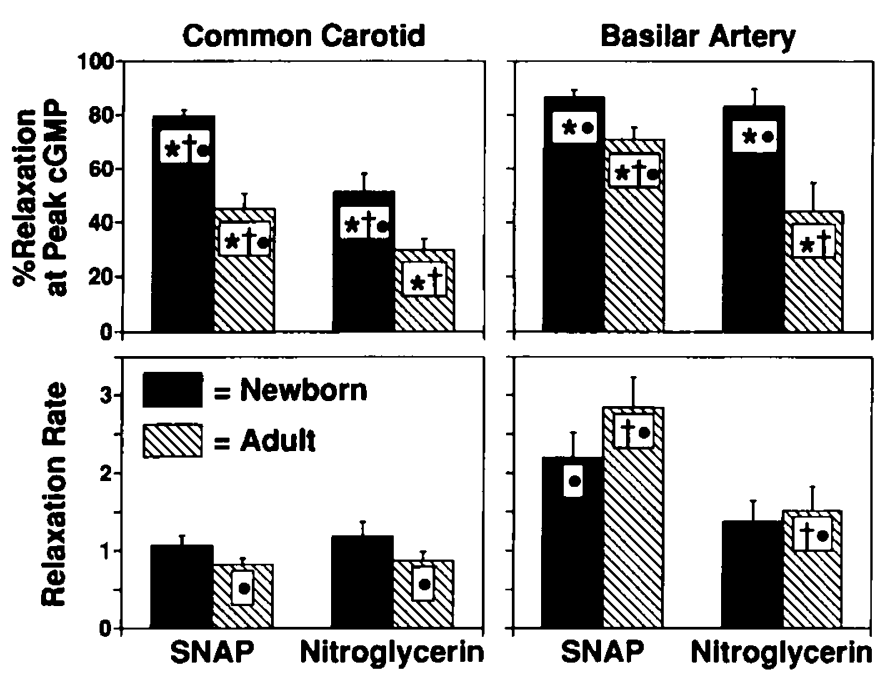

Figure 5. The upper panels show the relaxation responses observed at the times corresponding to peak cGMP in each group. The lower panels indicate the rate coefficients for relaxation in each group, obtained by fitting the relaxation-time data to a monoexponential decay model. All values are given as means and SEM. The numbers of animals used are as indicated in the previous legends. Statistically significant differences between groups are as indicated in the legend for Figure 2. mon carotid and basilar artery responses. Basilar arteries relaxed significantly faster than common carotid arteries in both newborns and adults, a finding probably related to differences in wall thickness and diffusional path length, as mentioned above. In association with these differences in relaxation rate, basilar arteries exhibited greater relaxation at peak cGMP than did corresponding common carotid arteries. Basilar arteries also had significantly greater basal levels of cGMP, suggesting that the balance between cGMP synthesis and degradation is tipped toward greater synthesis in the basilar arteries. Despite these and other minor differences, no significant differences in maximum efficacy or the effects of maturation thereupon were observed between basilar and common carotid arteries. This latter observation suggests that the influences governing changes in maximum efficacy are a generalized feature of maturation and are not specific for either intracranial or extracranial arteries. What these influences are and how they are coordinated with the myriad other changes accompanying maturation remain almost completely unexplored and thus constitute a promising and important topic for future investigations.

Acknowledgments. The authors thank Dr. John Fukuto of the UCLA Department of Pharmacology for the S-nitroso- $\mathrm{N}$-acetyl-penicillamine used in these studies. The authors also thank the Amersham Corporation of Arlington Heights, Illinois, for their cooperation during the validation studies of the cyclic nucleotide RIA.

\section{REFERENCES}

1. Pearce WJ, Hull AD, Long DM, Longo LD 1991 Developmental changes in ovine cerebral artery composition and reactivity. Am J Physiol 261:R458R465

2. Pape KE 1989 Etiology and pathogenesis of intraventricular hemorrhage in newborns. Pediatrics 84:382-385

3. Del Toro J, Louis PT, Goddard-Finegold J 1991 Cerebrovascular regulation and neonatal brain injury. Pediatr Neurol 7:3-12

4. Hayashi S, Park MK, Kuehl TJ 1984 Higher sensitivity of cerebral arteries isolated from premature and newborn baboons to adrenergic and cholinergic stimulation. Life Sci 35:253-260

5. Toda N, Shimizu I, Okamura T, Miyazaki M 1986 Age-dependent change in the response of isolated beagle cerebral arteries to vasoactive agents. J Cardiovasc Pharmacol 8:681-688

6. Wagerle LC, Kurth CD, Roth RA 1990 Sympathetic reactivity of cerebral arteries in developing fetal lamb and adult sheep. Am J Physiol 258:H1432 H1438

7. Murad F, Waldman S, Molina C, Bennett B, Leitman D 1987 Regulation and role of guanylate cyclase-cyclic GMP in vascular relaxation. Prog Clin Biol Res 249:65-76

8. Lincoln TM, Cornwell TL 1991 Towards an understanding of the mechanism of action of cyclic AMP and cyclic GMP in smooth muscle relaxation. Blood Vessels 28:129-137

9. Rand MJ 1992 Nitrergic transmission: nitric oxide as a mediator of nonadrenergic, non-cholinergic neuro-effector transmission. Clin Exp Pharmacol Physiol 19:147-169

10. Pelligrino DA, Koenig HM, Albrecht RF 1993 Nitric oxide synthesis and regional cerebral blood flow responses to hypercapnia and hypoxia in the rat. J Cereb Blood Flow Metab 13:80-87

11. Pearce WJ, Reynier Rebuffel AM, Lee J, Aubineau P, Ignarro L, Seylaz J 1990 Effects of methylene blue on hypoxic cerebral vasodilatation in the rabbit. J Pharmacol Exp Ther 254:616-625

12. Iwamoto J, Yoshinaga M, Yang SP, Krasney E, Krasney J 1992 Methylene blue inhibits hypoxic cerebral vasodilation in awake sheep. J Appl Physiol 73:2226-2232

13. Zellers TM, Vanhoutte PM 1991 Endothelium-dependent relaxations of piglet pulmonary arteries augment with maturation. Pediatr Res 30:176-180

14. Pearce WJ, Longo LD 1991 Developmental aspects of endothelial function. Semin Perinatol 15:40-48 
15. Longo LD, Hull AD, Long DM, Pearce WJ 1993 Cerebrovascular adaptations to high-altitude hypoxemia in fetal and adult sheep. Am J Physiol 264:R65$\mathrm{R} 72$

16. Pearce WJ, Ashwal S, Cuevas J 1989 Direct effects of graded hypoxia on intact and denuded rabbit cranial arteries. Am J Physiol 257:H824-H833

17. Abman SH, Chatfield BA, Rodman DM, Hall SL, McMurtry IF 1991 Maturational changes in endothelium-derived relaxing factor activity of ovine pulmonary arteries in vitro. Am J Physiol 260:L280-L285

18. Fratacci MD, Frostell CG, Chen TY, Wain JJ, Robinson DR, Zapol WM 1991 Inhaled nitric oxide. A selective pulmonary vasodilator of heparin-protamine vasoconstriction in sheep. Anesthesiology 75:990-999

19. Ignarro LJ, Lippton H, Edwards JC, Baricos WH, Hyman AL, Kadowitz PJ, Gruetter CA 1981 Mechanism of vascular smooth muscle relaxation by organic nitrates, nitrites, nitroprusside, and nitric oxide: evidence for the involvement of S-nitrosothiols as active intermediates. J Pharmacol Exp Ther 218:739-749

20. Brien JF, McLaughlin BE, Kobus SM, Kawamoto JH, Nakatsu K, Marks GS 1988 Mechanism of glyceryl trinitrate induced vasodilation. I. Relationship between drug biotransformation, tissue cyclic GMP elevation and relaxation of rabbit aorta. J Pharmacol Exp Ther 244:322-327

21. Schini V, Schoeffter P, Miller RC 1989 Effect of endothelium on basal and on stimulated accumulation and efflux of cyclic GMP in rat isolated aorta. Br J Pharmacol 97:853-865

22. Komas N, Lugnier C, Andriantsitohaina R, Stoclet JC 1991 Characterisation of cyclic nucleotide phosphodiesterases from rat mesenteric artery. Eur J Pharmacol 208:85-87

23. Pato MD, Kerc E 1987 Characterization of the smooth muscle phosphatases and study of their function. Prog Clin Biol Res 245:207-218

24. Wolfe L, Corbin JD, Francis SH 1989 Characterization of a novel isozyme of cGMP-dependent protein kinase from bovine aorta. J Biol Chem 264:7734-7741

25. Twort $\mathrm{CH}$, van Breemen C 1988 Cyclic guanosine monophosphate enhanced sequestration of $\mathrm{Ca}^{2+}$ by sarcoplasmic reticulum in vascular smooth muscle. Circ Res 62:961-964

26. Giembycz MA, Raeburn D 1991 Putative substrates for cyclic nucleotidedependent protein kinases and the control of airway smooth muscle tone. $\mathrm{J}$ Auton Pharmacol 11:365-398 